\title{
Final state interactions in the nuclear response at large momentum transfer
}

\author{
Omar Benhar \\ INFN, Sezione di Roma. I-00185 Roma, Italy \\ Dipartimento di Fisica, \\ "Sapienza" Università di Roma. I-00185 Roma, Italy
}

(Dated: June 7, 2018)

\begin{abstract}
The convolution approach, widely employed to describe final state interactions in the response of many-body systems, is derived from the expression of the nuclear response in the zeroth-order ladder approximation. Within this framework, the folding function, accounting for the effects of interactions between the struck particle and the spectator system, can be immediately related to the spectral function of particle states. The role of nucleon-nucleon correlations in determining the energy dependence is analyzed.
\end{abstract}

PACS numbers: 13.6.Le, 25.30Fj, 25.30Rw

\section{INTRODUCTION}

In the impulse approximation (IA) regime, the response of a many-body system to a probe delivering momentum $\mathbf{q}$ and energy $\omega, S(\mathbf{q}, \omega)$, can be directly related to the spectral function describing the energy and momentum distribution of its constituents [1].

The IA is based on the premise that, as the space resolution of the probe is $\sim|\mathbf{q}|^{-1}$, at large enough $|\mathbf{q}|$ (typically $|\mathbf{q}| \gg 2 \pi / d, d$ being the average separation between target constituents) the target is seen by the probe as a collection of individual particles. In addition, final state interactions (FSI) between the hit constituent and the spectators are assumed to be negligibly small.

Within the IA scenario, scattering off a many body system reduces to the incoherent sum of scattering processes involving the target constituents, the energy and momentum of which are distributed according to the spectral function.

The goal of extracting information on the target spectral function from the measured cross sections has been pursued in a variety of contexts. The momentum distribution of liquid ${ }^{4} \mathrm{He}$, obtained from neutron scattering data, has been extensively analyzed to determine the condensate fraction 2], while a number of studies of the nuclear electromagnetic response have been aimed at identifying high momentum components, induced by short range nucleon-nucleon (NN) correlations, in the target wave function (for a recent review of electron-nucleus scattering in the quasielastic sector, see Ref. [3]).

Comparison between the results of theoretical calculations and data has consistently shown that the IA scheme fails to explain the measured cross sections at fully quantitative level, thus suggesting that FSI play a significant role [4 7]. Clearcut evidence of the persistence of FSI effects at large momentum transfer has been also provided by theoretical studies of both nuclear matter [8] and the Bose hard-sphere system [9].

In view of the fact that FSI may largely obscure the connection between the target response and the underlying energy and momentum distribution, a quantitative understanding of their effects must be regarded as a pre- requisite for the extraction of the relevant dynamical information from the data.

In the widely employed convolution approach, $S(\mathbf{q}, \omega)$ is written in the form [4, 6 ]

$$
S(\mathbf{q}, \omega)=\int d \omega^{\prime} S_{0}\left(\mathbf{q}, \omega^{\prime}\right) F_{\mathbf{q}}\left(\omega-\omega^{\prime}\right),
$$

where $S_{0}(\mathbf{q}, \omega)$ denotes the response in the absence of FSI, the effects of which are described by the folding function $F_{\mathbf{q}}(\omega)$.

This article is aimed at showing that Eq.(11), often justified using heuristic arguments [10], can be obtained in a consistent fashion within a more fundamental approach, based on of many body theory and the spectral function formalism. [11, 12]. The proposed interpretation turns out to significantly affect the large $\omega$ behavior of the folding function, that in turn determines the tails of the measured inclusive cross section [6].

The expression of the nuclear response in terms of spectral functions is derived in Section $\amalg A$, while Section $\amalg \mathrm{IB}$ is devoted to the discussion of the eikonal approximation, employed to obtain the folding function of Eq. (11). The elements of the calculation of $F_{\mathbf{q}}(\omega)$ are analyzed in Section III. In order to illustrate the role of FSI in determining the electromagnetic nuclear response at large momentum transfer, in Section IV we report the results of theoretical calculations of the inclusive electron scattering cross section at $|\mathbf{q}| \gtrsim 2 \mathrm{GeV}$. Finally, In Section $\mathrm{V}$ we summarize our findings and state the conclusions.

\section{FORMALISM}

\section{A. Folding function and particle spectral function}

For simplicity, we will consider a scalar probe interacting with uniform (i.e. translationally invariant) isospin symmetric nuclear matter. The generalization to the case of finite nuclei and electromagnetic interactions does not involve any additional conceptual difficulties.

The response of the system can be written in terms of the imaginary part of the particle-hole propagator 
$\Pi(\mathbf{q}, \omega)$ according to 11,13

$$
S(\mathbf{q}, \omega)=\frac{1}{\pi} \operatorname{Im} \Pi(\mathbf{q}, \omega),
$$

with

$$
\Pi(\mathbf{q}, \omega)=\left\langle 0\left|\rho_{\mathbf{q}}^{\dagger} \frac{1}{H-E_{0}-\omega-i \epsilon} \rho_{\mathbf{q}}\right| 0\right\rangle,
$$

and $\epsilon=0^{+}$. The operator

$$
\rho_{\mathbf{q}}=\sum_{\mathbf{k}} a_{\mathbf{k}+\mathbf{q}}^{\dagger} a_{\mathbf{k}}
$$

$a_{\mathbf{k}}^{\dagger}$ and $a_{\mathbf{k}}$ being nucleon creation and annihilation operators, respectively, describes the fluctuation of the target density induced by the interaction with the probe, while the target ground state satisfies the Schrödinger equation

$$
H|0\rangle=E_{0}|0\rangle,
$$

where $H$ denotes the nuclear hamiltonian.

At large momentum transfer, the effects of long range correlations, arising from mixing of one particle-one hole states and leading to the excitation of collective modes, are expected to become negligible [14]. In this kinematical regime, the particle-hole propagator can be written in terms of the Green's functions $G_{h}(\mathbf{k}, E)$ and $G_{p}(\mathbf{k}, E)$, describing the propagation of a nucleon in a hole or particle state with momentum $\mathbf{k}$ and energy $E$.

The resulting expression of the response, often referred to as zeroth-order ladder approximation [13, 15], reads

$$
S(\mathbf{q}, \omega)=\int d^{3} k d E P_{h}(\mathbf{k}, E) P_{p}(\mathbf{k}+\mathbf{q}, \omega-E),
$$

where $P_{h}$ and $P_{p}$ denote the hole and particle spectral functions, respectively, simply related to the corresponding Green's functions through [13, 15]

$$
P_{h(p)}(\mathbf{k}, E)=-\frac{1}{\pi} \operatorname{Im} G_{h(p)}(\mathbf{k}, E) .
$$

The hole spectral function and the momentum distribution, defined as

$$
n(\mathbf{k})=\int d E P_{h}(\mathbf{k}, E),
$$

can be obtained within the framework of non relativistic many-body theory. Calculations of $P_{h}$ in isospin symmetric nuclear matter at equilibrium density have been carried out using both Correlated Basis Function (CBF) perturbation theory [16] and the self-consistent Green's function approach [17].

Within the IA scheme, in which FSI are disregarded, the particle spectral function is approximated with that of the non interacting Fermi gas. The resulting response reads

$$
\begin{aligned}
S_{0}(\mathbf{q}, \omega) & =\int d^{3} k d E P_{h}(\mathbf{k}, E) \theta\left(|\mathbf{k}+\mathbf{q}|-k_{F}\right) \\
& \times \delta\left(\omega-E-E_{\mathbf{k}+\mathbf{q}}\right),
\end{aligned}
$$

where $\theta(x)$ is the Heaviside step function, $k_{F}$ is the Fermi momentum and $E_{\mathbf{k}+\mathbf{q}}$ denotes the kinetic energy of a nucleon carrying momentum $\mathbf{k}+\mathbf{q}$.

While providing an excellent description of the measured nuclear cross sections in the region of the quasielastic peak, the IA scheme leads to largely underestimate the data at lower energy loss [3]. In the case of neutron scattering on liquid ${ }^{4} \mathrm{He}$, deviations from the IA predictions, occurring also at the quasielastic peak, make it difficult the identification of the delta function singularity associated with the condensate.

In inclusive processes, as long as the set of available final states is complete, FSI do not affect the total (i.e. $\omega$-integrated) cross section at fixed q. At large momentum transfer, their main effect is a broadening of the $\delta$ function appearing in Eq.(9), owing to the fact that the collisions between the struck particle and the spectators couple the one particle-one hole state produced at the primary interaction vertex to more complex final states. As a result, the state describing the struck particle acquires a finite lifetime $\tau \sim 1 / \rho \sigma$, where $\sigma$ is the total NN scattering cross section and $\rho$ is the target density.

The starting point of our derivation is the expression of the response of Eq. (6), providing a link between the particle spectral function and the folding function appearing in Eq. (11). Substituting Eq. (9) into Eq. (1) and comparing the result to the right hand side of Eq. (6) we find

$$
\begin{aligned}
& P_{p}(\mathbf{k}+\mathbf{q}, \omega-E)=\theta\left(|\mathbf{k}+\mathbf{q}|-k_{F}\right) \\
& \quad \times \int d \omega^{\prime} F_{\mathbf{q}}\left(\omega-\omega^{\prime}\right) \delta\left(\omega^{\prime}-E-E_{\mathbf{k}+\mathbf{q}}\right) .
\end{aligned}
$$

At large momentum transfer $\left(|\mathbf{q}| \gg 2 k_{F} \sim 500 \mathrm{MeV}\right.$ in electron-nucleus scattering), the condition that the momentum of the struck particle be larger than the Fermi momentum is always satisfied and the $\theta$-function can be omitted. Hence, approximating $\mathbf{k}+\mathbf{q} \approx \mathbf{q}$, which in turn implies $E_{\mathbf{k}+\mathbf{q}} \approx E_{\mathbf{q}}$, Eqs. (6) and (1) become equivalent if

$$
F_{\mathbf{q}}(\omega)=P_{p}\left(\mathbf{q}, \omega+E_{\mathbf{q}}\right) .
$$

Note that the folding function is defined in such a way as to peak at $\omega=0$, whereas the spectral function $P_{p}(\mathbf{q}, E)$ is peaked at $E=E_{\mathbf{q}}$. Furthermore, from Eq. (11) it follows that, since the spectral function is a positive quantity [13], the folding function $F_{\mathbf{q}}(\omega)$ is also positive.

At moderate momentum transfer, the hole and particle spectral functions can be consistently obtained using non relativistic many-body theory [13]. However, in the kinematical region of large momentum transfer the motion of the struck nucleon in the final state can no longer be described using the non relativistic formalism.

At IA level, the above problem can be easily circumvented replacing the non relativistic kinetic energy with its relativistic counterpart in Eq.(9). On the other hand, inclusion of FSI requires further approximations, needed to obtain the particle spectral function, or, equivalently, the folding function appearing in Eq.(1). 
A theoretical approach to calculate the folding function, based on a generalization of Glauber theory of high energy proton-nucleus scattering 18], has been developed in Ref. [6] and extensively applied to the analysis of the measured inclusive electron-nucleus cross sections [19, 20].

To make a connection between the formalism of Ref. [6] and the one based on spectral functions, in Section IIB we will outline the derivation of the Green's function of particle states within the eikonal approach.

\section{B. The eikonal approximation}

In order to keep contact with the formalism of nuclear many-body theory, in this Section we will use the non relativistic formalism. The generalization to the case of relativistic particles will be discussed at a later stage.

Let us first consider a nucleon scattering from the potential $V$. Its propagation is described by the Green's function

$$
G=\left(E-\mathbf{k}^{2} / 2 m-V+i \epsilon\right)^{-1},
$$

that can be obtained solving the integral equation

$$
G=G_{0}+G_{0} V G,
$$

the free space Green's function $G_{0}$ being given by

$$
G_{0}=\left(E-\mathbf{k}^{2} / 2 m+i \epsilon\right)^{-1} .
$$

The eikonal approximation is based on the tenet that, at high energy, the projectile particle travels along a straight trajectory with constant speed. Under this assumption, one can write $E \approx|\mathbf{p}|^{2} / 2 m, \mathbf{p}$ being the incident momentum, and the momentum in the intermediate states in the form

$$
\mathbf{k}=\mathbf{p}+\boldsymbol{\Delta}, \quad|\boldsymbol{\Delta}| \ll|\mathbf{p}| .
$$

Using the above relations and neglecting the term quadratic in $\boldsymbol{\Delta}$, Eq.(14) can be cast in the form

$$
G_{0}=\left[v\left(|\mathbf{p}|-k_{z}\right)+i \epsilon\right]^{-1},
$$

where $v=|\mathbf{p}| / m$ is the nucleon velocity and the $z$-axis has been chosen along the direction of $\mathbf{p}$. Fourier transformation to coordinate space yields

$$
\begin{aligned}
\left\langle\mathbf{r}^{\prime}\left|G_{0}\right| \mathbf{r}\right\rangle & =\int \frac{d^{3} k}{(2 \pi)^{3}} \frac{e^{i \mathbf{k} \cdot\left(\mathbf{r}^{\prime}-\mathbf{r}\right)}}{v\left(|\mathbf{p}|-k_{z}\right)+i \epsilon} \\
& =-\frac{i}{v} \delta\left(\mathbf{b}^{\prime}-\mathbf{b}\right) \theta\left(z^{\prime}-z\right) e^{i|\mathbf{p}|\left(z^{\prime}-z\right)},
\end{aligned}
$$

where $\mathbf{b}$ is the projection of $\mathbf{r}$ on the plane perpendicular to the momentum $\mathbf{p}$.

Substituting the above equation in Eq. (13) and assuming that the interaction $V$ be local, one readily obtains the coordinate-space Green's function

$$
\left\langle\mathbf{r}^{\prime}|G| \mathbf{r}\right\rangle=\left\langle\mathbf{r}^{\prime}\left|G_{0}\right| \mathbf{r}\right\rangle U\left(\mathbf{b}, z^{\prime}-z\right),
$$

with

$$
U\left(\mathbf{b}, z^{\prime}-z\right)=\exp \left[-\frac{i}{v} \int_{0}^{z^{\prime}-z} d \zeta V(\mathbf{b}, z+\zeta)\right],
$$

describing the motion of a particle moving along the straight trajectory $\mathbf{r}(\tau)=\mathbf{r}+\mathbf{v} \tau$, constrained by the condition $\mathbf{r}(t)=\mathbf{r}^{\prime} \equiv\left(\mathbf{b}, z^{\prime}\right)$ at time $t=\left(z^{\prime}-z\right) / v$.

The above result can be used to obtain the scattering wave function, $\psi_{\mathbf{p}}$, from the equation

$$
\psi_{\mathbf{p}}=(\mathbb{1}+G V) \phi_{\mathbf{p}},
$$

where $\phi_{\mathbf{p}}$ is an eigenfunction of the free hamiltonian, i.e. a plane-wave of momentum $\mathbf{p}$. The resulting wave function can be written in the form

$$
\psi_{\mathbf{p}}(\mathbf{b}, z)=e^{i p z}\left[1-\int_{-\infty}^{z} d z^{\prime} \Gamma_{\mathbf{p}}\left(\mathbf{b}, z^{\prime}\right)\right],
$$

with

$$
\Gamma_{\mathbf{p}}(\mathbf{b}, z)=V(\mathbf{b}, z) \exp \left[-\frac{i}{v} \int_{-\infty}^{z} d z^{\prime} V\left(\mathbf{b}, z^{\prime}\right)\right] .
$$

Using the above expression of $\psi_{\mathbf{p}}$ and the definition of the scattering amplitude at incident momentum $\mathbf{p}$ and momentum transfer $\mathbf{k}=\mathbf{p}-\mathbf{p}^{\prime}$ (see, e.g., Ref. 21])

$$
f_{\mathbf{p}}(\mathbf{k})=-\frac{m}{2 \pi}\left\langle\phi_{\mathbf{p}^{\prime}}|V| \psi_{\mathbf{p}}\right\rangle
$$

one finds that the quantity defined in Eq.(21) is trivially related to $f_{\mathbf{p}}(\mathbf{k})$ through Fourier transformation, i.e.

$$
\Gamma_{\mathbf{p}}(\mathbf{r})=-\frac{2 \pi}{m} \int \frac{d^{3} k}{(2 \pi)^{3}} e^{-i \mathbf{k} \cdot \mathbf{r}} f_{\mathbf{p}}(\mathbf{k}) .
$$

In the case of a nucleon propagating through nuclear matter in the aftermath of the interaction with an external probe, the eikonal approximation must be supplemented with the further assumption that the configuration of the spectator system be frozen, i.e. do not change due to interactions with the fast projectile particle.

The eikonal factor including all contributions arising from collisions involving the projectile particle, labelled by the index 1 , and the $N-1$ target nucleons takes the form

$$
\exp \left[-\frac{i}{v} \int_{0}^{z} d \zeta \sum_{j=2}^{N} v_{1 j}\left(\mathbf{r}_{1}+\hat{\mathbf{z}} \zeta-\mathbf{r}_{j}\right)\right],
$$

where $v_{i j}$ is the bare NN potential and $\hat{\mathbf{z}}$ denotes the unit vector along the $z$-axis.

Expanding the exponential appearing in the righthand side of Eq.(24), one obtains a series, the terms of which are associated with processes involving an increasing number of interactions between the projectile particle and the spectator nucleons. The terms corresponding to repeated interactions with the same spectator can be collected and summed up to all orders by replacing the bare 
$v_{i j}$ with the coordinate-space scattering amplitude $\Gamma_{\mathbf{p}}$ of Eq.(23).

Average over the nuclear matter ground state leads to the final expression of the eikonal factor [compare to Eq.(18) ]

$$
U(z)=\exp \left[-\frac{i}{v} \int_{0}^{z} d \zeta V(\zeta)\right]
$$

with

$$
V(\zeta)=\left\langle 0\left|\sum_{j=2}^{N} \Gamma_{\mathbf{p}}\left(\mathbf{r}_{1 j}+\hat{\mathbf{z}} \zeta\right)\right| 0\right\rangle
$$

Note that the right hand side of the above equation involves an average over the degrees of freedom of both the projectile particle and the spectators. In general, averaging over the position of the struck particle at $\tau=0$, $\mathbf{r}_{1}$, amounts to a further approximation. However, owing to translation invariance, in uniform nuclear matter this is not the case.

The particle spectral function can be readily computed using Eq. (7) and the Green's function obtained within the eikonal approximation (for simplicity, from now on the subscript $p$, specifying the particle part of both the Green's function and the associated spectral function, will be omitted)

$$
\begin{aligned}
G(\mathbf{b}, z) & =-\frac{i}{v} \delta(\mathbf{b}) \theta(z) \\
& \times \exp \left[i p z-\frac{i}{v} \int_{0}^{z} d \zeta V(\zeta)\right],
\end{aligned}
$$

with $V(\zeta)$ given by Eq.(26).

\section{CALCULATION OF THE PARTICLE SPECTRAL FUNCTION}

\section{A. NN scattering amplitude}

At high incident momentum, $\mathbf{p}$, the NN scattering amplitude of Eq. (22) extracted from the measured cross sections is usually written in terms of three parameters, in the form

$$
f_{p}(\mathbf{k})=\frac{p}{4 \pi} \sigma_{p}\left(\alpha_{p}+i\right) e^{-\beta_{p} \mathbf{k}^{2}},
$$

where $\mathbf{k}$ is the momentum transfer and $\sigma_{p}$ is the total cross section, while $\alpha_{p}$ and $\beta_{p}$ describe the ratio between real and imaginary part and the slope, respectively. Note that the above expression fulfills the optical theorem, stating that the forward scattering amplitude satisfies the relation

$$
\operatorname{Im} f_{p}(0)=\frac{p}{4 \pi} \sigma_{p}
$$

by construction.
The main effect of FSI, i.e. the broadening of the $\delta$ function appearing in Eq.(9), arises from the imaginary part of the scattering amplitude, while the real part produces a shift of the response of the order of $10 \mathrm{MeV}$ at most. As we are focusing on a kinematical region in which the typical scale of the energy transfer is several hundreds $\mathrm{MeV}$, in the following the effect of the real part of the scattering amplitude will be disregarded, setting $\alpha_{p}=0$ in Eq. 28.

Medium modifications of the NN scattering cross section are expected to be important, and must be taken into account. In Ref. [23], the relation between NN scattering in vacuum and in nuclear matter has been analyzed under the assumption that the nuclear medium mainly affects the flux of incoming particles and the phase space available to final state particles, while leaving the transition probability unchanged.

The phase space of elastic NN collisions is reduced by Pauli blocking, whereas the modification of the flux is due to the fact that the nucleons involved in the scattering process are bound, and therefore off the mass-shell. Within the approach of Ref. 23], this feature is taken into account by replacing the bare nucleon mass with a momentum dependent effective mass.

The numerical results reported in this article have been obtained using the parametrization of the NN scattering amplitude of Eq.(28), with values of $\beta_{p}$ and $\sigma_{p}$ taken from the fit of Refs. [24, 25]. The total cross sections have been corrected for medium effects according to the generalization of the procedure of Ref. [23] described in Ref. [26]. As shown in Fig. 1, the resulting total cross sections are significantly reduced, with respect to the free space values. At beam energies $\gtrsim 800 \mathrm{MeV}$ the ratio between the proton-neutron cross sections in medium and in vacuum turns out to be $\sim 0.8$, and largely energy independent.

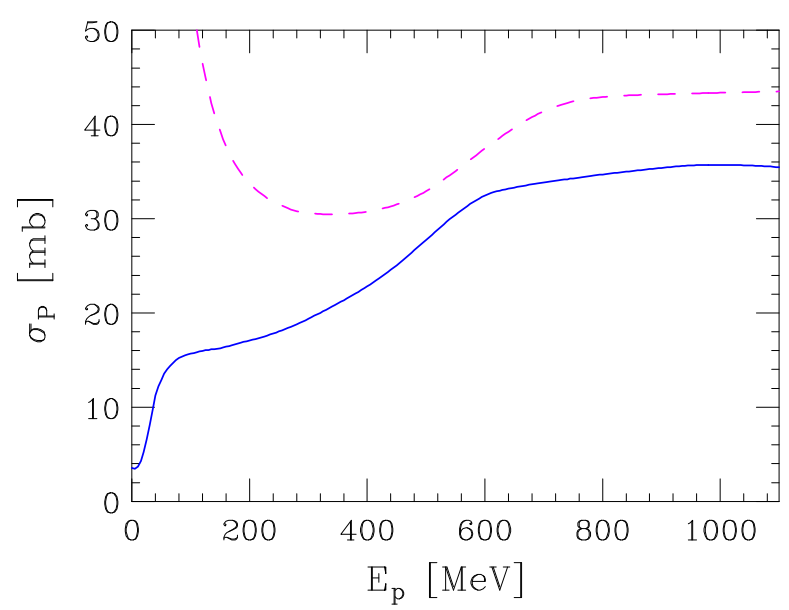

FIG. 1: (color online) Total proton-neutron cross section as a function of the projectile kinetic energy in the Lab frame. The dashed line shows the free space cross section, while the solid line has been obtained including medium modifications according to the procedure described in Refs. 23, 26]. 


\section{B. Eikonal factor}

Using the expression of the scattering amplitude discussed in the previous Section, one can compute the eikonal factor of Eq.(25) with the interaction defined by Eq.(26). The ground state expectation value appearing in the right hand side can be cast in the form

$$
V(\zeta)=\int d^{3} r g(r) \Gamma_{p}(\mathbf{r}+\hat{\mathbf{z}} \zeta)
$$

where $g(r)$ is the pair distribution function, yielding the probability of finding two nucleons separated by a distance $r$ in the nuclear matter ground state.

The behavior of $g(r)$ is dictated by the strong dynamical NN correlations induced by nuclear forces, as well as by the weaker statistical correlations due to Pauli's exclusion principle. It the absence of all correlations $g(r) \equiv 1$. Realistic calculations of the nuclear matter pair distribution function have been carried out within the Fermi-Hyper-Netted-Chain (FHNC) approach [27]. Figure 2 shows a comparison between the results of Ref. 27] and the pair distribution function of the non interacting Fermi gas.

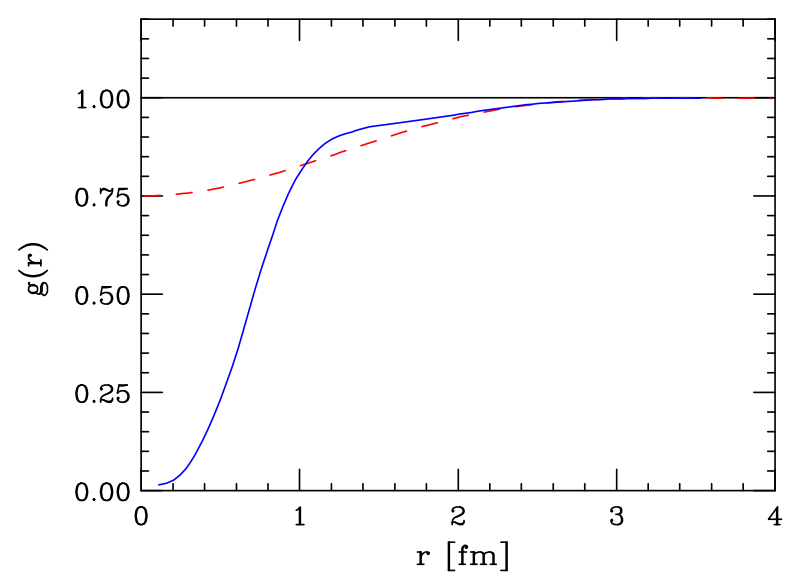

FIG. 2: (color online) Spin-isospin averaged NN pair correlation function in isospin symmetric nuclear matter at equilibrium density. The solid curve shows the result of the manybody calculation of Ref. [27], based on a realistic nuclear hamiltonian, while the dashed curve takes into account statistical correlations only. The reference line $g(r) \equiv 1$ corresponds to the quasiparticle approximation discussed in the text, in which all correlations are neglected.

Note that in the simple case of a zero-range coordinatespace interaction, and neglecting correlations altogether, Eq.(30) yields

$$
\operatorname{Im} V=-\frac{1}{2} \rho v \sigma_{p},
$$

independent of $\zeta$. Note, however, that $\operatorname{Im} V$ depends on density, a feature that turns out to be very important in the applications to light nuclei.

\section{Spectral function}

Using the results of the previous Sections, the particle spectral function can be obtained from Eq.(77), yielding

$$
P(\mathbf{p}, E)=-\frac{1}{\pi} \operatorname{Im} \frac{i}{v} \int_{0}^{\infty} d z e^{i\left(k_{z}-p\right) z-\frac{i}{v} \int_{0}^{z} d \zeta V(\zeta)},
$$

where $p=|\mathbf{p}|$. Under the assumption that $V$ be purely imaginary, and defining

$$
W(t)=-\frac{1}{t} \int_{0}^{t} d \tau \operatorname{Im} V(v \tau),
$$

where $\tau=\zeta / v$, Eq.(32) can be rewritten

$$
P(\mathbf{p}, E)=\frac{1}{\pi} \int_{0}^{\infty} d t \cos \left[v\left(k_{z}-p\right) t\right] e^{-W(t) t} .
$$

Note that the right hand side of the above equation depends on the energy $E=E_{\mathbf{k}}$ through the momentum $\mathbf{k}$, while $W(t)$ depends on $p$ through the momentumdependence of the NN scattering amplitude.

The spectral function takes a very simple form in the case of uncorrelated nucleons and zero-range scattering amplitude. From Eqs. (31) and (33) it follows that, in this case,

$$
W=\frac{1}{2} \rho v \sigma_{p},
$$

independent of $t$, and

$$
P(\mathbf{p}, E)=\frac{1}{\pi} \frac{W}{\left[v\left(k_{z}-p\right)\right]^{2}+W^{2}} .
$$

\section{Effects of NN correlations}

The expression of the spectral function of Eq.(36) deserves some comments. Under the assumptions discussed in Section IB $v\left(k_{z}-p\right) \approx E_{\mathbf{k}}-E_{\mathbf{p}}$, and Eq.(36) can be rewritten in a form reminiscent of the definition of the spectral function in terms of the nucleon self energy $\Sigma(\mathbf{p}, E)[28]$

$$
P(\mathbf{p}, E)=\frac{1}{\pi} \frac{\operatorname{Im} \Sigma(\mathbf{p}, E)}{\left[E-E_{\mathbf{p}}-\operatorname{Re} \Sigma(\mathbf{p}, E)\right]^{2}+[\operatorname{Im} \Sigma(\mathbf{p}, E)]^{2}} .
$$

Comparison between the above equation and Eq. (36) shows that assuming a purely imaginary NN scattering amplitude and neglecting all correlations amounts to approximating the self energy with its energy-independent low-density limit, given by the forward NN scattering amplitude [29, 30], implying

$$
\operatorname{Re} \Sigma(\mathbf{p}, E)=0 \quad, \quad \operatorname{Im} \Sigma(\mathbf{p}, E)=\frac{1}{2} \rho v \sigma_{p} .
$$

The spectral function obtained within the above approximation includes the effect of collisions between a nucleon 
carrying momentum $\mathbf{p}$ and the nucleons belonging to the Fermi sea. This scheme may be regarded as a quasiparticle approximation, in which only the pole contribution to the Green's function is taken into account 31. However, the contribution of $\mathrm{NN}$ correlations, resulting in an explicitly energy-dependent self energy, is disregarded altogether.

Within the formalism discussed in this work, the energy dependence associated with correlation effects arises from the time dependence of the function $W(t)$, defined by Eq.(33), which is in turn to be ascribed to the timedependence of the radial distribution function.

To see this, consider the simple case of a zero-range coordinate-space interaction, i.e.

$$
\operatorname{Im} \Gamma_{p}(\mathbf{r})=-\frac{1}{2} \rho v \sigma_{p} \delta(\mathbf{r}) .
$$

Substituting the above expression in Eq. (30) and using Eq.(33), one finds the result

$$
W(t)=\frac{1}{2} \rho v \sigma_{p} \frac{1}{t} \int_{0}^{t} d \tau g(v \tau),
$$

which reduces to the time-independent form of Eq.(35) for $g(v \tau) \equiv 1$. Note that within the eikonal approximation time and distance travelled by the projectile particle are trivially related, as the velocity, $v$, is assumed to be constant.

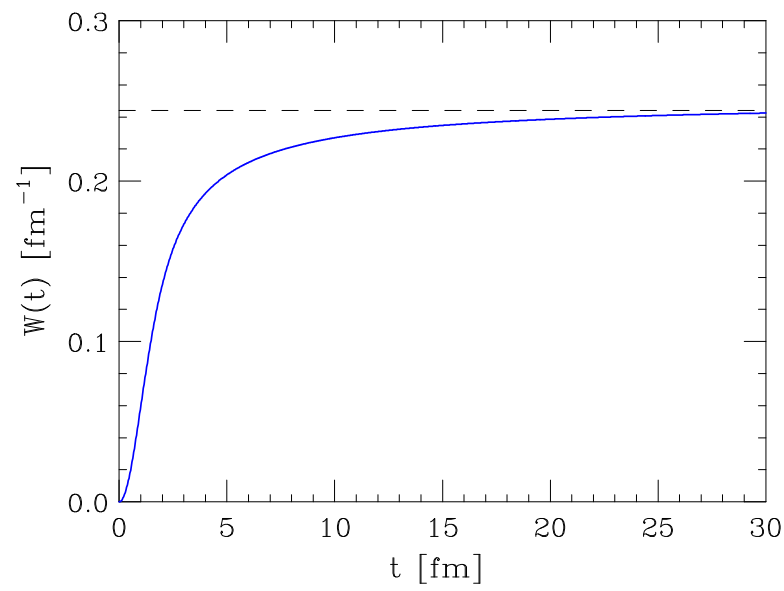

FIG. 3: (color online) Time-dependence of the function $W(t)$, defined by Eq. (40), computed using the radial distribution function of Fig. 2 and medium modified NN cross sections obtained from the procedure described in Refs. [23, 26]. The nucleon momentum has been set to $p=1 \mathrm{GeV}$.

The shape of the function $\mathrm{W}(\mathrm{t})$, obtained from the above equation using the nuclear matter radial distribution function displayed in Fig. 2 and medium modified NN cross sections is illustrated in Fig. 3 for the case of a nucleon carrying momentum $p=1 \mathrm{GeV}$. The deviation from the asymptotic value reflects the fact that, owing to the short-range repulsive core of the NN interaction, the struck particle is surrounded by a correlation hole, that makes the probability of FSI at short $t$ vanishingly small. As a consequence NN correlations mostly affect the high-energy behavior of the spectral function.

\section{RESULTS}

In this section we report the results of the application of the formalism discussed in the previous Sections to the study of the electromagnetic response of isospin symmetric nuclear matter at momentum transfer $|\mathbf{q}| \gtrsim 1 \mathrm{GeV}$.

The eikonal approximation derived in the previous Sections to calculate the particle spectral function, yielding in turn the folding function $F_{\mathbf{q}}(\omega)$, can be readily extended to the relativistic regime, relevant to the kinematical region under consideration. It has been shown (see, e.g., Refs. 32, 33]) that, under the assumptions discussed in Section IIB, the relativistic propagator can be linearized and interactions lead to a phase-shift of the plane wave describing the motion of the projectile particle. The form of the eikonal phase turns out to be the same as the one given by Eq. (25).

The double differential cross section of the process

$$
e+A \rightarrow e^{\prime}+X
$$

including the effect of FSI, is obtained convoluting the IA result with the folding function of Eq. (11) according to

$$
\frac{d \sigma}{d \Omega d \omega}=\int d \omega^{\prime}\left(\frac{d \sigma}{d \Omega d \omega}\right)_{I A} F_{\mathbf{q}}\left(\omega-\omega^{\prime}\right) .
$$

The details of the calculation of the IA cross section within the formalism of nuclear many body theory can be found, e.g., in Ref. [3].

As an example, Fig. 4 shows the behavior of the folding function for incident momentum $\sim 2 \mathrm{GeV}$, computed using the radial distribution function of Fig. 2 and medium modified NN cross sections. For simplicity, we have neglected the effect of the finite range of the NN scattering amplitude, which is known to be small [6], setting $\beta_{p}=0$ in Eq. (28). The solid and dashed lines correspond to the full result and to the quasiparticle approximation [see Eq. [38)], in which NN correlation are neglected altogether. It clearly appears that the inclusion of correlations leads to a reduction of the tails of the folding function, resulting in turn in a reduction of FSI effects in the low- $\omega$ tail of the differential cross section.

These features can be observed in Fig. 5, showing the cross section of isospin symmetric nuclear matter at beam energy $E_{e}=3.6 \mathrm{GeV}$ and electron scattering angle $\theta_{e}=30 \mathrm{deg}$. Comparison between the solid and dashed lines, corresponding to the full and IA calculations, respectively, clearly shows that in the region of low energy loss FSI provide the dominant contribution, which brings theoretical results to agree with the extrapolated 


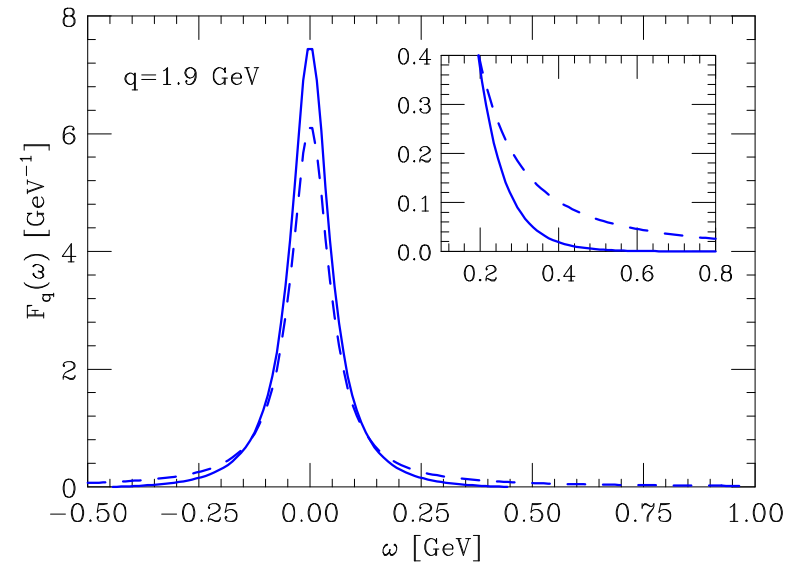

FIG. 4: (color online) Energy dependence of the folding function defined in Eq. (11). The solid and dashed line correspond to the full calculation and to the quasiparticle approximation of Eq. (38), respectively. The calculations have been carried out for isospin symmetric nuclear matter at equilibrium density. The nucleon momentum $|\mathbf{q}|=1.9 \mathrm{GeV}$ corresponds to quasi free kinematics at incident energy $E_{e}=3.6 \mathrm{GeV}$ and electron scattering angle $\theta_{e}=30 \mathrm{deg}$.

data of Ref. 34. The role of NN correlations is illustrated by the dot-dash line, obtained using the folding function computed within the quasiparticle approximation (dashed line of Fig. 4). It is apparent that neglecting correlations leads to largely overestimate FSI effects.

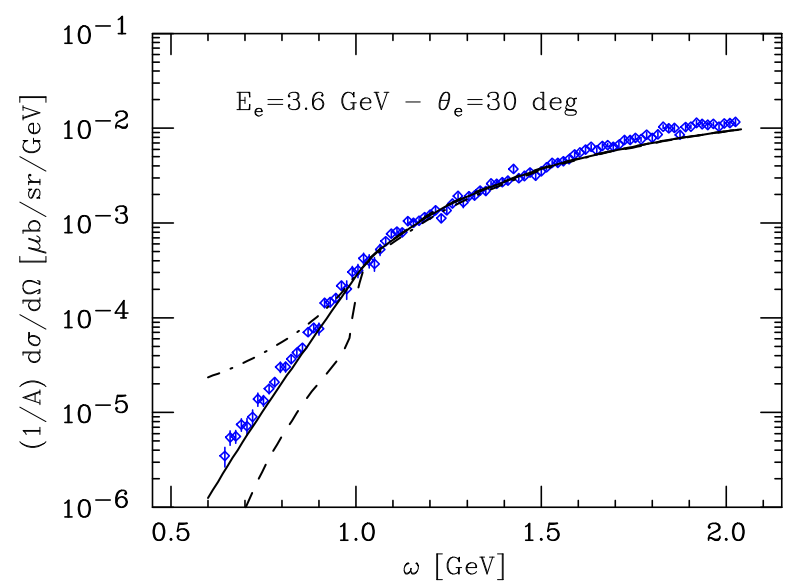

FIG. 5: (color online) Differential cross section of the scattering process $e+A \rightarrow e^{\prime}+X$ on isospin symmetric nuclear matter, at beam energy $E_{e}=3.6 \mathrm{GeV}$ and electron scattering angle $\theta_{e}=30 \mathrm{deg}$. The solid and dot-dash lines represent the results of the full calculation and those obtained within the quasiparticle approximation discussed in the text, respectively. The cross section obtained within the IA, i.e. neglecting FSI, is displayed by the dashed line. The data points show the extrapolated nuclear matter cross section of Ref. 34].
In Fig. 6] the differential cross section obtained using the formalism discussed in this article is compared to the extrapolated nuclear matter data of Ref. 34] and to the ${ }^{56} \mathrm{Fe}$ data of Ref. [35] at beam energy $E_{e}=4 \mathrm{GeV}$ and electron scattering angle $\theta_{e}=30 \mathrm{deg}$. The proposed approach appears to provide a quantitative description of the measured cross sections over a range exceeding five orders of magnitude.

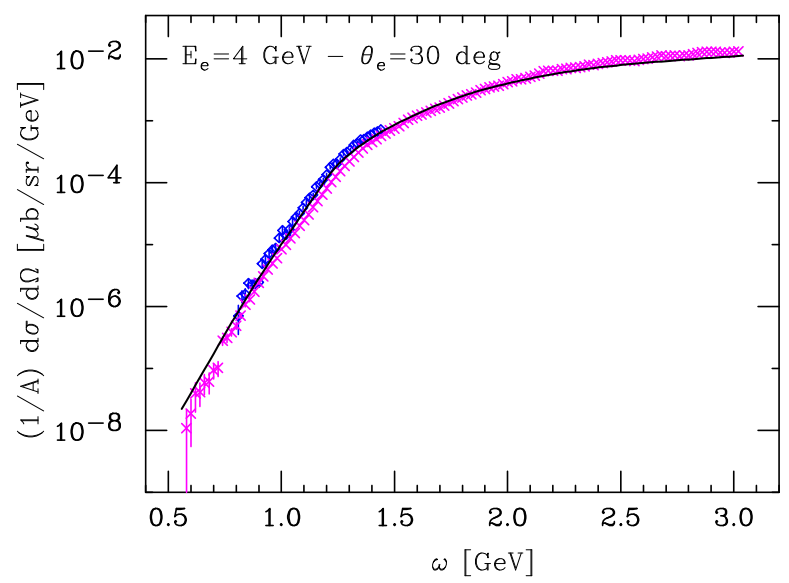

FIG. 6: (color online) Differential cross section of the scattering process $e+A \rightarrow e^{\prime}+X$ on isospin symmetric nuclear matter, at beam energy $E_{e}=4 \mathrm{GeV}$ and electron scattering angle $\theta_{e}=30 \mathrm{deg}$. The solid line shows the results of the full calculation, including FSI. The diamonds corresponds to the extrapolated nuclear matter cross section of Ref. [34]. For comparison, the crosses also show the cross section of Ref. [35], measured in the same kinematical setup using a ${ }^{56} \mathrm{Fe}$ target.

\section{CONCLUSIONS}

We have discussed the description of FSI in the nuclear response, and shown that the widely employed convolution form of Eq. (1) can be obtained from a fundamental approach based on nuclear many-body theory, using the spectral function formalism.

The folding function of the convolution approach turns out to be directly related to the spectral function describing high momentum nucleons occupying particle states, which can be calculated within the eikonal approximation. The main elements entering this calculation are the measured NN scattering cross sections, modified to take into account the effects of the nuclear medium, and the radial distribution function $g(r)$, yielding the probability of finding two nucleons separated by a distance $r$ in the nuclear ground state. Both the nucleon effective mass, driving the modifications of the NN cross section, and the radial distribution function are obtained from accurate many-body calculations based on a realistic nuclear hamiltonian. 
The interpretation of the folding function as a particle spectral function allows one to unambiguously identify the contribution of NN correlations, arising from the energy dependence of the nucleon self-energy.

The main effect of FSI is a redistribution of the strength, producing a slight decrease of the response in the region of the quasi free peak and a sharp enhancement of its tails. Inclusion of NN correlations, resulting in the appearance of the energy dependence of the nucleon self-energy, leads to a substantial reduction of FSI, to be ascribed to the correlation hole surrounding the struck nucleon.

The results of numerical calculations of the electronnucleus scattering cross section at momentum transfer $\gtrsim 2 \mathrm{GeV}$ show that FSI are the dominant reaction mechanism in the region of low energy loss, corresponding to values of the Bjorken scaling variable $x \gtrsim 1.5$. Their effect brings the theoretical results into agreement with the data over a broad range of energy loss.

A pioneering study of the nuclear matter cross section within the convolution approach was carried out in the 1990s [6]. The results of this work suggested that, even after inclusion of NN correlations, using the free space NN cross section to compute the folding function leads to sizably overestimate FSI. The authors of Ref. [6] argued that the source of this problem could be traced back to modifications of the NN cross section arising from the internal structure of the nucleon, and advocated the occurrence color transparency to explain the disagreement between theory and data.

In the present work we have taken into account the modifications of the free space NN cross sections arising from many-body effects, that lead to a decrease of FSI. Moreover, unlike that of Ref. [6], the folding function resulting from the approach described in this article is non-negative by definition. The occurrence of oscillations in the tails of the folding function of Ref. [6], associated with the appearance of negative values, is incompatible with the interpretation in terms of particle spectral function, and as such unphysical. In fact, it must be regarded as numerical noise.

A comprehensive analysis of the large database of inclusive data at large momentum transfer within the present approach will provide valuable information on the dependence of FSI on both the nuclear mass number, $A$, and the squared four momentum transfer, $Q^{2}$. Such a study may help to shed light on the interpretation of the measured ratios of inclusive nuclear cross sections at $x>1$ [36, 37].

\section{Acknowledgments}

This work was partially supported by INFN, under grant MB31, and MIUR PRIN, under grant "Many-body theory of nuclear systems and implications on the physics of neutron stars". The author is deeply indebted to Ingo Sick for a number of illuminating discussions on issues related to the subject of this article, as well as for a critical reading of the manuscript.
[1] O. Benhar, A. Fabrocini, and S. Fantoni, Phys. Rev. Lett. 87, 052501 (2001).

[2] H.A. Mook, Phys. Rev. B 37, 5806 (1988).

[3] O. Benhar, D. Day, and I. Sick, Rev. Mod. Phys. 80, 189 (2008).

[4] T.R. Sosnick, W.M. Snow, R.N. Silver, and P.E. Sokol, Phys. Rev. B 43, 216 (1991).

[5] H. Meier-Hajduk, Ch. Hajduk, P.U. Sauer, and W. Theis, Nucl. Phys. A395, 332 (1983).

[6] O. Benhar, A. Fabrocini, S. Fantoni, G.A. Miller, V.R. Pandharipande, and I. Sick, Phys. Rev. C 44, 2328 (1991).

[7] C. Ciofi degli Atti, D.B. Day, and S. Liuti, Phys. Rev. C 46, 1045 (1992).

[8] O. Benhar, Phys. Rev. Lett. 83, 3130 (1999).

[9] J.J. Weinstein and J.W. Negele, Phys. Rev. Lett. 49, 1016 (1982).

[10] P.C. Hohenberg, and P.M. Platzman, Phys. Rev. 152, 198 (1966).

[11] G.E. Brown, Many body physics (North Holland, Amsterdam, 1972)

[12] A. Fetter, and J. Walecka, Quantum theory of many particle systems (McGraw-Hill, New York, 1971)

[13] O. Benhar, A. Fabrocini, and S. Fantoni, Nucl. Phys. A550, 201 (1992).

[14] O. Benhar, and N. Farina, Phys. Lett. B 680, 305 (2009).
[15] W.H. Dickhoff, and C. Barbieri, Prog. Part. Nucl. Phys. 52 (2004) 377.

[16] O. Benhar, A. Fabrocini, and S. Fantoni, Nucl. Phys. A505, 267 (1989).

[17] A. Ramos, A. Polls, and W.H. Dickhoff, Nucl. Phys. A503, 1 (1989).

[18] R. Glauber, in Lectures in Theoretical Physics, Eds. W.E. Brittin and L.G. Dunham (Wiley, New York, 1959), Vol. 1, p. 315.

[19] O. Benhar, and V.R. Pandharipande, Phys. Rev. C 47, 2218 (1993)

[20] O. Benhar, A. Fabrocini, S. Fantoni, and I. Sick, Nucl. Phys. A579, 493 (1994).

[21] A.G. Sitenko, Scattering Theory (Springer, Berlin, 1991).

[22] M. Petraki, E. Mavrommatis, O. Benhar, J.W. Clark, A. Fabrocini, and S. Fantoni, Phys. Rev. C 67 (2003) 014605.

[23] V.R. Pandharipande and S.C. Pieper, Phys. Rev. C 45, 791 (1992).

[24] T.G. O'Neill et al., Phys. Lett. B351, 87 (1995)

[25] T.G. O'Neill, private communication

[26] O. Benhar, A. Fabrocini, S. Fantoni, V.R. Pandharipande, S.C. Pieper, and I. Sick, Phys. Lett. B359, 8 (1995).

[27] R. Schiavilla, D.S. Lewart, V.R. Pandharipande, R.B. Wiringa, and S. Fantoni, Nucl. Phys. A473, 267 (1987). 
[28] J.P. Jeukenne, A. Lejeune, and C. Mahaux, Phys, Rep. 2, 83 (1976).

[29] C.B. Dover, J. Hüfner, and L.H. Lemmer, Ann. Phys. (N.Y.) 66, 248 (1971).

[30] J. Hüfner, Phys. Rep. 21, 1 (1975).

[31] O. Benhar, A. Fabrocini, and S. Fantoni, Phys. Rev. 41, R24 (1990).

[32] S.J. Wallace and J.A. McNeil, Phys. Rev. D 16, 3565 (1977).
[33] D. Debruyne, J. Ryckebusch, W. Van Nespen, and J. Janssen, Phis. Rev C 62, 024611 (2000).

[34] D. Day et al., Phys. Rev. C 40, 1011 (1989).

[35] J. Arrington et al., Phys. Rev. Lett 82, 2056 (1999).

[36] O. Benhar, A. Fabrocini, S. Fantoni, and I. Sick, Phys. Lett. B343, 47 (1995).

[37] N. Fomin et al., Phys. Rev. Lett. 108, 092502 (2012). 